\title{
Competencia intercultural en el ámbito de las drogodependencias
}

\section{Intercultural competence in the area of drug dependencies}

\author{
Javier ARZA PORRAS \\ Universidad Pública de Navarra, España \\ jarvier.arza@unavarra.es \\ José CARRÓN SÁNCHEZ \\ Universidad de Zaragoza, España \\ jcarron@unizar.es
}

Recibido: $27 / 06 / 2015$

Revisado: 22/07/2015

Aceptado: 11/11/2015

Disponible on line: 20/01/2016

\section{Resumen}

En este artículo partimos de una concepción multidimensional o biopsicosocial de las drogodependencias. Dentro de este marco de análisis, abogamos por una mayor visibilización de la dimensión intercultural, ya que es imprescindible para el diseño y desarrollo de procesos de intervención integrales. Proponemos la competencia intercultural como un modelo de trabajo que puede permitir el incremento en las capacidades de instituciones y profesionales, especialmente relevante en el caso del Trabajo Social, para abordar eficazmente la dimensión intercultural aludida. Tras una amplia revisión de la literatura científica, hemos definido cinco procesos que pueden contribuir a reforzar la competencia intercultural de una institución y cuatro procesos que pueden contribuir a incrementar la competencia intercultural de un o una profesional. Aunque han sido seleccionados para su aplicación en el ámbito de las drogodependencias, todos estos procesos también pueden servir para mejorar la atención a cualquier otro tipo de persona o grupo culturalmente diverso.

Palabras clave: drogodependencias, interculturalidad, competencia intercultural, educación entre iguales, antropología de la salud.

\begin{abstract}
This article takes a multidimensional or biopsychosocial conception of drug dependency as its starting point. Within this analytical framework, we advocate making the intercultural dimension more visible, since it is essential for the design and implementation of integral intervention processes. We propose intercultural competence as a working model that can increase the capacities of institutions and professionals - a particularly important consideration in the case of social workin order to effectively address the aforementioned cultural dimension. After an extensive review of the scientific literature, we have defined five processes that can contribute to strengthening an institution's intercultural competence and four processes that can do the same for a professional's intercultural competence. Though selected for application in the area of drug dependencies, all these processes can also prove useful in improving attention to any other kind of culturally diverse group or person.
\end{abstract}

Keywords: drug dependency, interculturality, intercultural competence, peer education, anthropology of health.

Referencia normalizada: Arza Porras, J. y Carrón Sánchez, J. (2016): «Competencia intercultural en el ámbito de las drogodependencias». Cuadernos de Trabajo Social, 29(1): 109-118.

Sumario: Introducción. 1. Consumo de drogas, drogodependencia y cultura. 2. Concepto de competencia intercultural. 3. Competencia intercultural institucional. 4. Competencia intercultural profesional. 5. A modo de conclusión. 6. Referencias bibliográficas.

\section{Introducción}

La competencia intercultural es un modelo de trabajo que pretende mejorar la atención a personas con identidades culturales diferentes, facili- tando que profesionales e instituciones integren una serie de conocimientos, actitudes y habilidades que lo hagan posible. Es, por lo tanto, un modelo de comprensión y acción fundamental para 
una disciplina como Trabajo Social, en la que es una constante la interacción con personas y grupos con modelos de referencia diferentes.

Tradicionalmente se aplica en la intervención con la población inmigrante o con minorías étnicas autóctonas. Sin embargo, el valor añadido de nuestra propuesta reside en que se propone su aplicación a la mejora de la atención a otros colectivos con expresiones identitarias no étnicas, como es el caso de las personas drogodependientes.

Asumir la dimensión intercultural en este ámbito supone profundizar en la comprensión de las drogodependencias como un fenómeno multidimensional, alejándose de aquellas visiones y abordajes reduccionistas que limitan la comprensión del fenómeno a la conducta del individuo que decide tomar drogas o no tomarlas aislado de cualquier factor individual, social o farmacológico. Hasta el momento, el modelo que se ha aproximado más a esa comprensión multidimensional ha sido el biopsicosocial ${ }^{1}$. Sin embargo, y aunque formalmente ese modelo ha contemplado el contexto cultural dentro del amplio cajón de la dimensión social, lo cierto es que lo cultural ha sido relegado enormemente hasta el momento, tanto en las investigaciones como en el diseño de las políticas o en la práctica cotidiana de los y las profesionales. En ese sentido, el desarrollo de la competencia intercultural puede facilitar el diseño y desarrollo de políticas y procesos de atención más integrales, que tengan en cuenta la influencia del conjunto de ideas, rituales, creencias o significados que, tanto las personas drogodependientes como los y las profesionales o la sociedad, construyen en torno al consumo de drogas y la drogodependencia.

En definitiva, la competencia intercultural permite visibilizar la dimensión social de las drogodependencias, contribuyendo de esta manera a contrarrestar la tendencia sanitarista observada en los últimos años. En ese sentido, representa una oportunidad para seguir reivindicando la interdisciplinariedad y, específicamente, la presencia necesaria de profesionales del Trabajo Social en los equipos.

\section{Consumo de drogas, drogodependencia y cultura}

Como cualquier otro fenómeno relacionado con la salud-enfermedad, el consumo de drogas y la drogodependencia son conceptos socialmente construidos. Elster, señala que «el condicionamiento químico es esencial para iniciar la adicción, pero la verdadera ansia adictiva se conforma a través de un estilo de vida adictivo» (citado en Comas, 2005, p. 19). Un estilo de vida, o «cultura de las ansias adictivas químicas» (ídem), que se construye en grupo, pero también en permanente interacción con las interpretaciones sociales sobre las drogas y sus usos, y con las circunstancias socio-económicas del contexto. Un ejemplo clásico al respecto lo constituye el síndrome de abstinencia por la heroína. En la década de 1980, varios especialistas en tratamiento de las personas con drogodependencia describieron cómo algunos heroinómanos jóvenes, con un historial de consumo no muy prolongado de dosis bajas y con un grado de pureza de la heroína muy reducido, presentaban cuadros espectaculares de síndrome de abstinencia. En la interpretación de este hecho se barajaba que el síndrome experimentado no respondía al grado de dependencia física, sino a la codificación cultural que de ese comportamiento se realizaba en su grupo de iguales. Además, esa codificación conectaba con la representación social mayoritaria, alimentada fuertemente por unos medios de comunicación que presentaban continuamente escenas de síndromes de abstinencia (en películas y reportajes) incluso desde antes de la extensión del consumo de heroína en España (Comas, 1986). Al margen de este ejemplo concreto, en la literatura vinculada a la antropología de la salud (Menéndez, 1998; Romaní, 2002) se ha argumentado ampliamente que, además de la composición química o el contexto socio-económico, en los efectos de las drogas tienen un gran peso determinados elementos codificados culturalmente por el grupo de referencia: las expectativas de consumo, la función del consumo e incluso hábitos o rituales como la manera de obtener la sustancia, la vía o el contexto del consumo, la dosis o el grado de pureza.

${ }^{1}$ Desde la década de 1980, el biopsicosocial ha sido el sistema o modelo de creencias asumido formalmente en el marco de las políticas sobre drogodependencias en el Estado español, pero también en Europa. Se puede ver un análisis pormenorizado en torno a la evolución de los modelos de creencias, y su influencia en el diseño y desarrollo de las políticas, en Arza y Carrón (2014). 
Pero ¿qué supondría incorporar la perspectiva cultural en la atención a las drogodependencias? Fundamentalmente que se tenga en cuenta, tanto en los diagnósticos como en los tratamientos o en el diseño de las políticas, la relevancia de aspectos como: la influencia del grupo de referencia en los hábitos y rituales de consumo; en la interpretación del consumo; en la interpretación de los riesgos vinculados al consumo; en las prácticas de auto-atención para reducir el riesgo; y evitar de este modo los daños en la auto-representación como consumidor de drogas y/o drogodependiente; en la delimitación y valoración de qué es un problema de consumo y cuándo aparece; en la definición de cuándo, cómo y dónde se pide ayuda y en la construcción de la relación de ayuda y/o terapéutica. En definitiva, se trataría de integrar en el proceso de atención a la dimensión illnes $^{2}$ y de tener en cuenta que las personas drogodependientes también poseen saberes y prácticas de auto-atención (sector popular, según la clasificación de Kleinman) ${ }^{3}$.

No obstante, en realidad el tipo de perspectiva no debe ser únicamente cultural, sino que tiene que ser intercultural. El análisis no debe cir- cunscribirse a la observación de los elementos culturales vinculados a las personas usuarias y sus grupos de referencia, sino que debe ampliarse al análisis de la interrelación entre éstos y los modelos de creencias sobre las drogodependencias preponderantes en el contexto social en general, pero también en el profesional del Trabajo Social (o en el de cualquier otro miembro del equipo) que construye la relación de ayuda y/o terapéutica con el sujeto en concreto. A ese respecto, por ejemplo, la mirada que prevalezca en relación con las personas drogodependientes influirá en el tipo de relación que se construya. Lógicamente no es lo mismo que la institución o el/la trabajador social les observe como experimentadores curiosos que lo haga como personas enfermas o como delincuentes o como pecadores o como ciudadanos o como débiles o como egocéntricos o como irresponsables o como personas con capacidades para sobrevivir en contextos hostiles o como personas sin autocontrol ${ }^{4}$. Cada una de estas miradas (y otras que podríamos añadir) cuenta con un modelo de creencias, con una cultura respecto a las drogas y las drogodependencias que las sustenta. Una cultura

2 Como una manera de afirmar la diversidad de concepciones en torno a la salud y la enfermedad, la antropología de la salud distingue tres dimensiones (Fabrega, 1974; Comelles y Martínez, 1993; Canals, 2004):

- Dimensión disease. Responde a la definición de salud y enfermedad, pretendidamente objetiva, realizada por el sistema de saberes y prácticas reconocido en una sociedad determinada (la medicina científica, en la nuestra).

- Dimensión illness. Es la vivencia subjetiva de la salud y la enfermedad que tiene un determinado grupo o una determinada persona. Esta vivencia puede coincidir o no con la dimensión disease.

— Dimensión sickness. Son las ideas socialmente aceptadas en torno a la salud y la enfermedad. Pueden estar más o menos influidas por la dimensión disease o por la dimensión illness.

Esta tipología permite visualizar la necesidad de tener en cuenta las diversas interpretaciones y los diferentes discursos que pueden existir sobre la salud y la enfermedad. La insuficiente sensibilidad y habilidad (competencia) de las redes de recursos profesionales para dialogar con las dimensiones illnes y sickness puede provocar importantes barreras en el encuentro con determinados grupos de personas usuarias.

3 Según Kleinman (1980), en cada sociedad o grupo cultural interactúan tres sectores en la construcción de los sistemas de atención a la salud-enfermedad:

- Sector popular. Está compuesto por la auto-atención y el tratamiento no profesionalizado ni especializado por parte de personas del entorno más cercano.

- Sector profesional. Está compuesto por el conjunto de profesionales y procedimientos legitimados en una sociedad.

- Sector folk. En este sector se engloba el conjunto de prácticas terapéuticas que no siguen los parámetros admitidos por la medicina científica: curanderos, sanadores, chamanes, astrólogos, terapeutas alternativos, etc.

Uno de los elementos fundamentales de esta clasificación nos muestra que todo grupo social posee saberes y prácticas de respuesta a la salud y la enfermedad. Más allá de considerarlos correctos o erróneos es preciso que el sector profesional ponga en marcha procesos para entenderlos e integrarlos en una respuesta conjunta del profesional con el grupo de personas afectadas.

4 Estas mismas y diferentes miradas pueden observarse en la auto-representación como consumidor de drogas y/o drogodependiente, y también deben considerarse en la construcción de la relación de ayuda y/o terapéutica. 
respecto a un fenómeno (el consumo de drogas y la drogodependencia) que moviliza emociones (e incluso pasiones) al mismo nivel que otros fenómenos sociales como el sexo o la muerte. El consumo de drogas y la drogodependencia nos remiten al miedo a lo desconocido, y especialmente al miedo a la pérdida del control. Como señalan Romaní y Rekalde (2002):

La construcción del — problema de la droga - ha creado otro - chivo expiatorio-, que como muchos otros - malos-, llámense éstos herejes, judíos, brujas, pobres, locos, etc., confirma a los - buenos - la justeza de su comportamiento y situación (ique tanto les ha costado conseguir!), ayudando así a superar las dudas que pudieran tener, $\mathrm{y}$ tendiendo a justificar y reforzar un determinado orden social (p. 15).

En el caso de la población usuaria o potencialmente usuaria de la red de tratamiento para drogodependientes, la situación actual de incremento de la diversidad de perfiles implica también una gran pluralidad de elementos culturales vinculados al consumo de drogas. En ese sentido es aún más importante la sensibilidad intercultural del profesional del Trabajo Social, el esfuerzo por entender el modo de mirar la realidad del otro, o mejor dicho, de cada uno de los otros. Precisamente el concepto de competencia intercultural, al que nos referiremos a partir del siguiente apartado, surge para subrayar la importancia de incorporar todos esos conocimientos, actitudes y habilidades, a las competencias que todo profesional debe manejar y que toda institución debe integrar.

\section{Concepto de competencia intercultural}

El concepto de competencia cultural surge en la literatura científica, a finales de la década de 1970 en Estados Unidos e Inglaterra, como respuesta a la percepción de dificultades del encuentro entre la población inmigrante y los diferentes sistemas de prestación de servicios. No obstante, su mayor desarrollo se ha producido en el ámbito sanitario estadounidense. En ese contexto, uno de los conceptos que se han manejado ha sido el siguiente: «La competencia cultural es la integración compleja de conocimiento, actitudes y habilidades que aumenta la comunicación entre culturas diferentes y las interacciones apropiadas/efectivas con los otros» (American Academy of Nursing, citado en Lillo Crespo, Vizcaya Moreno, Domínguez Santamaría y Galao Malo, 2004, p. 2). No obstan- te el análisis de otras definiciones complementarias nos permitirá observar la estrecha vinculación entre la competencia cultural y los principios fundamentales del Trabajo Social, como pueden ser: la necesidad de vincularse con el contexto del otro, el compromiso con los menos favorecidos, el empoderamiento o la participación. Así, Campinha Bacote resalta que la competencia cultural no es un estado que se alcanza, sino un «proceso en el cual los profesionales del cuidado de la salud continuamente se esfuerzan por conseguir la habilidad y la disponibilidad para trabajar efectivamente dentro del contexto cultural de la familia, el individuo, o la comunidad» (citado en OsorioMerchán y Lucero, 2008, p. 3). Por otro lado, Papadopolus incluye en su concepto la responsabilidad del o de la profesional en la defensa de las personas menos favorecidas y en su empoderamiento para que puedan participar en la definición de sus tratamientos y en la defensa de sus derechos (citado en Osorio-Merchán y Lucero, 2008). Oriol Romaní (2002), aunque sin referirse específicamente a la competencia cultural, resalta la importancia de hallar puntos de encuentro entre la visión del mundo de las personas usuarias y la de los y las profesionales. Nos recuerda que «la asistencia no es sólo un asunto técnico, sino un conjunto de técnicas, rituales aplicados y complejos procesos de movilización que se enmarcan en una determinada visión del mundo con sus correspondientes sistemas normativos») (p. 10). Continuando con esta idea, Vázquez (2002) resalta el valor de la negociación de los significados culturales de los hechos, entendiendo lo que representan y su interpretación desde el marco cultural de la persona usuaria. Finalmente, Fernández Liria y Pérez Sales (2011) apuestan por anteponer al concepto cultural el prefijo inter, para resaltar la idea del encuentro y la relación entre dos culturas o cosmovisiones, la de la persona usuaria y la de los y las profesionales, que también son seres culturales.

Habitualmente se considera que la competencia intercultural consiste únicamente en la incorporación de una serie de conocimientos, actitudes $\mathrm{y}$ habilidades en los y las profesionales, para facilitar una mejor atención a las personas con cosmovisiones o culturas diversas. Podemos denominar a ese tipo de competencia intercultural profesional. Sin embargo, también podemos hablar de competencia intercultural institucional. En ese caso, la competencia intercultural consis- 
te en que las instituciones (un centro de salud, un centro de servicios sociales, una asociación o un hospital) incorporen prácticas (de formación y selección de personal, de acogida o de adaptación en los protocolos) que faciliten el acceso y la atención de calidad a las personas de culturas diversas. Aunque gran parte de las propuestas y análisis que hemos podido encontrar en la literatura científica se refieren fundamentalmente al primer tipo de competencia, en este artículo plantearemos también algunas áreas para el desarrollo de la competencia intercultural institucional ${ }^{5}$.

\section{Competencia intercultural institucional}

En este apartado describiremos los procesos que consideramos prioritarios para el desarrollo de la competencia intercultural en una institución que trabaje en el ámbito de las drogodependencias (Cuadro 1). No obstante, todos estos procesos también pueden servir para mejorar la atención prestada a cualquier otro tipo de persona o grupo culturalmente diverso.

Para la selección y descripción de los cinco procesos propuestos hemos tenido en cuenta diversas fuentes: la revisión de la literatura científica, determinadas conclusiones extraídas de una evaluación de las políticas sobre drogodependencias realizada por los autores de este artículo ${ }^{6}$

\section{Procesos de competencia intercultural institucional}

— Selección de personal y composición de los equipos.

- Formación del personal.

- Detección-atención proactiva y adaptada de necesidades.

- Alianza con la comunidad.

- Modelo participativo para la definición de normas y pautas de funcionamiento.

Cuadro 1. Procesos de competencia intercultural institucional. $y$, en tercer lugar, nuestra propia experiencia como profesionales en este ámbito.

\subsection{Selección de personal y composición de los equipos}

Una institución contará con mayor competencia intercultural cuando en su equipo se integran profesionales que representan las distintas identidades culturales que conforman su población objetivo. Este proceso de competencia intercultural institucional guarda una estrecha relación con la denominada estrategia de apoyo entre iguales. Disponer de iguales en el equipo (peer worker, según la terminología empleada por algunos autores y autoras) le permite a la institución mejorar la intensidad y calidad de su proximidad a la población objetivo, con lo que se puede mejorar la cobertura, incrementar la integralidad de los diagnósticos y de los planes de tratamiento, aumentar la identificación de las personas usuarias con el tratamiento y mejorar los resultados. Esta práctica ha sido impulsada principalmente por profesionales del Trabajo Social en los programas de intervención con población inmigrante y con la comunidad gitana, pero en otros campos específicamente socio-sanitarios las experiencias son mucho más reducidas y puntuales. En el ámbito de las drogodependencias, la presencia de ex-drogodependientes en los equipos de tratamiento ha sido habitual en los modelos de comunidad terapéutica que se extendieron a finales de la década de 1970. Incluso algunas organizaciones, como El Patriarca, Reto, Remar, Betel y otros centros similares, contaban con equipos compuestos únicamente por personas que habían sido tratadas previamente en el mismo centro. El fuerte cuestionamiento a estos modelos de tratamiento que se produjo durante la profesionalización de la atención a las drogodependencias (fundamentalmente a finales de la década de 1980) ha podido influir en que las experiencias de incorporación de peer worker en este ámbito hayan sido reducidas (especialmente en el sector público). Sin embargo algunas organizaciones privadas, como

5 En algunas tipologías también se contempla un tercer nivel de competencia intercultural: la competencia estructural. Se refiere al desarrollo de políticas o estrategias estructurales que disminuyan las barreras que pueden tener las minorías culturales para acceder a las oportunidades. Este tercer nivel no ha sido abordado en este artículo, aunque supone un interesante y necesario ámbito de trabajo para futuras investigaciones.

6 Evaluación del I Plan de Drogodependencias de Navarra (1993-2009). Fue utilizada para la elaboración del II Plan. Una de las debilidades detectadas en este trabajo estaba vinculada al tipo de actitudes de los y las profesionales con las personas drogodependientes. En la evaluación se consideraba que esta debilidad estaba muy relacionada con los modelos de creencias profesionales y que influía de manera muy relevante en la relación terapéutica o de ayuda. 
Proyecto Hombre o Dianova, cuentan actualmente en sus equipos con profesionales que fueron tratados en sus programas de rehabilitación. No obstante, y a diferencia de las experiencias de los años 1970 y 1980, estos peer worker conviven en los equipos con otros y otras profesionales tradicionales y deben pasar por un completo y prolongado programa de capacitación ${ }^{7}$.

\subsection{Formación del personal}

Una institución contará con mayor competencia intercultural cuando su equipo cuenta con una formación adecuada en este terreno. El tipo de formación que se propone debe orientarse a desarrollar competencias cognitivas, actitudinales y procedimentales que permitan mejorar la calidad de la atención a todo tipo de personas usuarias, sean cuales sean sus identidades culturales. Por ejemplo:

Aspectos relacionados con la cultura, como podrían ser la propia concepción de la salud o la enfermedad, las variaciones en la forma de expresar los síntomas, las atribuciones de los mismos, la reflexión acerca de los referentes culturales del modelo biomédico occidental, el estudio de los posibles factores psicosociales relacionados con la salud, las diferentes maneras de buscar cuidados sanitarios,... la exploración de las preconcepciones culturales y étnicas de uno mismo» (Fernández Liria y Pérez Sales, 2011, p. 55).

En profesiones, como el Trabajo Social, es imprescindible que estos contenidos estén incluidos en la formación de Grado. Además, también es fundamental que formen parte de las ofertas formativas de postgrado y de formación continua.

\subsection{Detección-atención proactiva y adaptada de necesidades}

Cuando se trabaja con grupos culturales es importante que las instituciones asuman una actitud proactiva en la detección de necesidades y en su atención. De otra manera, refugiarse en que se ofrecen los mismos servicios a toda la población, sin discriminación alguna, en realidad puede conllevar la aparición de desigualdades reales y el mantenimiento de necesidades y problemáticas ocultas para los servicios sociosanitarios.

Una de las estrategias que puede facilitar esta detección proactiva de necesidades ya ha sido comentada: el apoyo entre iguales; y otra estrategia puede ser la alianza con las organizaciones que representan al grupo cultural, como comentaremos más adelante. Por otro lado, los programas asertivos comunitarios y otras estrategias de proximidad, como la educación de calle o los programas de reducción de daños, también pueden favorecer la detección y atención proactiva y adaptada de necesidades. Todas estas estrategias de proximidad cuentan con unos principios estrechamente vinculados al ADN del Trabajo Social: trabajo en el propio medio de las personas usuarias, atención integral e individualizada, empoderamiento y acompañamiento (Arza y Carrón (2014). Otra de las estrategias consiste en flexibilizar y adaptar los protocolos de atención a las necesidades y condicionantes de las personas usuarias. Algunas áreas fundamentales de adaptación pueden ser las siguientes:

- La institución debe contar con procedimientos establecidos que le permitan detectar y posteriormente corregir las barreras en la accesibilidad (horarias, de ubicación geográfica, de diseño de las infraestructuras, etc.).

- La institución debe asegurarse de manera proactiva que sus distintas poblaciones objetivo conozcan adecuadamente los servicios ofrecidos y la manera de acceder a ellos.

- La institución debe disponer de procedimientos que le permitan detectar las necesidades específicas de atención presentes en determinados grupos. Tras la detección, deben ponerse en marcha los mecanismos de adaptación en el tipo de itinerario de atención, en la duración y/o frecuencia de las atenciones, en el tipo de profesionales que atienden, etc.

\subsection{Alianza con la comunidad y, especial- mente, con las asociaciones representativas de la población atendida}

En el caso del consumo de drogas y las drogodependencias, las asociaciones han surgido mayoritariamente a iniciativa de familiares y otras personas sensibilizadas con esta problemática, limitándose las personas afectadas a ser usuarias de los servicios ofrecidos y beneficiarias de las acciones emprendidas. Sin embargo, podemos mencionar algunas experiencias que fomentan el protagonismo de las personas afectadas, como

${ }^{7}$ Un análisis sobre las posibilidades de aplicación de las estrategias peer worker para la intervención social puede ser encontrado en Arza y Carrón (2014). 
pueden ser los grupos de alcohólicos anónimos $\mathrm{y}$ alcohólicos rehabilitados que, aunque fundamentalmente son grupos de ayuda mutua, también realizan algunas actividades como grupos de interés y presión. También es reseñable la actividad de las diferentes asociaciones cannábicas, muy focalizadas en la defensa de los derechos de las personas consumidoras de esta sustancia y en la reivindicación de su legalización. Respecto al asociacionismo de personas usuarias de otro tipo de drogas ilegales, las experiencias en el Estado español son muy embrionarias. Los primeros grupos surgieron en Cataluña a finales de la década de 1990 y siempre muy vinculados a programas de reducción de daños. En la actualidad existen algunas experiencias puntuales en otras comunidades autónomas, pero no ha logrado consolidarse todavía un sólido movimiento de grupos de este tipo. No es ésta la experiencia de otros países europeos, donde asociaciones, como las Junkiebond holandesas, ASUD Francia o la Unión de Usuarios de Drogas de Dinamarca han jugado un rol muy relevante en la puesta en marcha de diferentes iniciativas en Europa de reducción de daños y en la normalización de este tipo de enfoque.

\subsection{Modelo participativo para la definición de normas y pautas de funcionamiento}

En nuestra sociedad la definición de las normas y pautas de funcionamiento del sistema sanitario (y del resto de sistemas) se realiza mayoritariamente a partir de las propuestas del sector profesional y atendiendo fundamentalmente a la dimensión disease. Sin embargo, como señala Oriol Romaní (2002):

Una intervención socio-sanitaria con pretensiones de efectividad tiene que estar de acuerdo, por lo menos hasta cierto nivel, con la visión del mundo de la gente asistida... Precisamente en las sociedades contemporáneas nos encontramos a menudo que la ausencia de estos mundos compartidos dificulta desde las relaciones médico-paciente hasta el desarrollo de los grandes programas socio-sanitarios» (p. 10).

En ese sentido, una institución interculturalmente competente es aquella que opera con un modelo participativo que incorpora el conjunto de visiones, necesidades, características y condicionantes de la población con la que trabaja, y define con las personas usuarias y otros agentes comunitarios los problemas prioritarios y los criterios de abordaje.

En el caso de las drogodependencias, el estigma social que soporta esta población, y la extendida representación social que identifica a sus miembros como personas incapaces de responsabilizarse y defender sus derechos, dificulta enormemente el desarrollo de este proceso. En ese sentido, un cambio de actitud en la institución y sus profesionales es imprescindible para que se pueda producir una participación real de la población usuaria.

En relación con este proceso, Fernández Liria y Pérez Sales señalan que «la participación debería incluirse desde la planificación de las atenciones hasta el control y evaluación de éstas, de modo que la práctica asistencial y de cuidados se aleje de cualquier riesgo de dominación y pasividad de los usuarios»». Asimismo, sostienen que «una primera aproximación, actualmente legalmente obligada, consiste en la recogida y tratamiento de las quejas y reclamaciones... las encuestas de satisfacción y opinión... Un nivel más sofisticado todavía, lo constituyen los grupos focales o foros de participación, de instituciones, informadores clave o usuarios» (p. 36).

\section{Competencia intercultural profesional}

Tal y como se reflejaba en el concepto ya referido de la American Academy of Nursing, la competencia intercultural integra conocimientos, actitudes y habilidades; es decir, posee una dimensión cognitiva, una dimensión afectiva y una dimensión instrumental. Por ello, los procesos de competencia profesional que hemos seleccionado están vinculados con esas tres dimensiones ${ }^{8}$ (Cuadro 2). Son procesos imprescindibles para el desarrollo del Trabajo Social, aunque lógicamente también lo son para el ejercicio de otras profesiones de ayuda. Son procesos que, como decíamos en el apartado 3.2., deben incorporarse a la formación de Grado, pero también al Posgrado y la formación continua.

\subsection{Conciencia intercultural crítica}

Un o una profesional con competencia intercultural debe tener un alto grado de conciencia de la in-

8 Para la selección y descripción de los cuatro procesos propuestos hemos utilizado las mismas fuentes señaladas en el apartado sobre competencia institucional. 


\section{Procesos de competencia intercultural profesional}

- Conciencia intercultural crítica.

- Conocimiento intercultural.

- Sensibilidad intercultural.

- Habilidad intercultural.

Cuadro 2. Procesos de competencia intercultural profesional.

fluencia de los elementos culturales en los comportamientos y las interacciones humanas. Debe reconocer sus propios valores, creencias y costumbres, para poder ser consciente de la influencia que tienen en la percepción y la valoración de las creencias, las costumbres y los valores de otras personas o grupos, e incluso en la conformación de prejuicios sobre otras identidades culturales. Como señala Malik (2009): «ello implica desarrollar la conciencia cultural crítica, o habilidad para evaluar de forma crítica y con criterios explícitos las perspectivas, costumbres y producciones de la propia cultura y de las demás» (p. 16).

\subsection{Conocimiento intercultural}

La dimensión cognitiva también tiene importancia en el desarrollo de la competencia intercultural. Un profesional, varón o mujer, con competencia intercultural debe tener amplios conocimientos sobre el concepto de cultura y, en este caso, sobre la influencia de los factores culturales en la salud (Lillo Crespo, Vizcaya Moreno, Domínguez Santamaría y Galao Malo, 2004; Boladeras, 2007; Osorio-Merchán y Lucero, 2008; Fernández Liria y Pérez Sales, 2011) y, según nuestra experiencia, también especialmente en el consumo de drogas y la drogodependencia. No obstante, es importante que los conocimientos previos sobre la identidad cultural de un grupo concreto no eviten que indaguemos sobre los valores, las creencias, los significados y las costumbres de cada uno de los individuos con quienes trabajamos ${ }^{9}$.

\subsection{Sensibilidad intercultural}

La sensibilidad intercultural forma parte de la dimensión afectiva de la competencia intercultural. De hecho, diferentes autores y autoras consi- deran que los aspectos actitudinales que la conforman constituyen la base de la competencia intercultural (Boladeras, 2007; Malik, 2009). Por ejemplo, Boladeras señala que la investigación indica que el trato desigual recibido por individuos pertenecientes a grupos minoritarios no suele responder a una falta de conocimiento o habilidades por parte del o de la profesional, sino a los estereotipos o actitudes discriminatorias que muestra con ese tipo de personas usuarias.

Vilà (2006) afirma que la dimensión afectiva o sensibilidad intercultural hace «referencia a aquellas capacidades de emitir respuestas emocionales positivas y controlar aquellas emociones que pueden perjudicar el proceso comunicativo intercultural» (p. 355). Siguiendo en parte las aportaciones de esta autora, describiremos a continuación los principales elementos que conforman la sensibilidad intercultural:

- Curiosidad, apertura y pasión por el encuentro con el otro diferente.

- Interés por aprender en la interacción intercultural.

- Sentimiento de comodidad y disfrute ante la interacción intercultural.

- Apertura hacia lo inesperado.

- Disposición para comprender la perspectiva del otro y, aunque no la comparta, apreciarla y respetarla.

- Perspectivismo, es decir, disposición a relativizar los valores, las creencias y las costumbres propias, para cambiar de perspectiva y para introducirse en la perspectiva del otro. En ese sentido, la sensibilidad intercultural implica conciencia intercultural y desarrollo de la capacidad empática.

- Disposición a reconstruir permanentemente la propia identidad a partir del contacto intercultural.

\subsection{Habilidad intercultural}

Las habilidades interculturales forman parte de la dimensión instrumental. La comunicación es una de las habilidades fundamentales en esta área. Qureshi Burckhardt, Revollo, Collazos, Visiers Würth y El Harrak (2009) plantean que el reto de la comunicación intercultural es doble:

\footnotetext{
9 Recordemos que la cultura es compartida diferencialmente. Variables como la edad, el sexo, la posición socioeconómica, la clase social o la religión, influyen en que cada grupo, subgrupo e individuo, viva la cultura común de forma diferenciada, dándole su tono particular.
} 
El primero es indirecto, o sea, no se debe a la comunicación en sí, sino a las asunciones más o menos automáticas que el personal sanitario y el paciente tienen de que no solamente son diferentes, sino que a veces son incompatibles (p. 57).

En ese sentido, el o la profesional con competencia intercultural debe saber trabajar sus expectativas ante el encuentro con la persona usuaria, desarrollando su habilidad para auto-examinar críticamente sus prejuicios y controlar la influencia de los mismos en la relación terapéutica o de ayuda. Asimismo, debe considerar las expectativas y prejuicios que la persona usuaria puede tener ante el encuentro con el o la profesional. Para ello, la acogida debe ser cuidada especialmente, desarrollando habilidades de comunicación verbal y no verbal que contribuyan a desactivar posibles expectativas negativas en la persona usuaria. El segundo reto, siguiendo la propuesta de estos autores, es directo y tiene que ver con las diferencias de estilos de comunicación y de interpretaciones de los significados. En este caso, las competencias son muy diversas:

- Capacidad para reconocer y adaptarse a pautas de comunicación diferentes: «expresiones faciales, contacto visual, uso del tacto, el lenguaje corporal y las prácticas de distancia y proximidad» (Osorio-Merchán y Lucero, 2008, p. 3).

- Habilidad para incrementar el conocimiento sobre la identidad cultural de la persona usuaria, sabiendo preguntarle adecuadamente sobre sus creencias, valores y comportamientos.

- Habilidad para entender los valores, las creencias y los comportamientos de la persona usuaria, desde su propia perspectiva.

- Expectativas adecuadas ante la comunicación intercultural. Capacidad para manejarse en ciertos niveles de ambigüedad y de entendimiento parcial.

Otra habilidad, estrechamente vinculada con la conciencia intercultural y el perspectivismo, es la capacidad de «descentración cultural». Según Cohen-Emerique (2000) esta habilidad supone:

Concienciarse de las referencias culturales propias y el poder distanciarse de ellas para lograr relativizar los puntos de vista, para alcanzar cierta neutralidad cultural que no es sinónimo de la negación de la identidad propia, sino que, al contrario, supone un reconocimiento de control sobre la propia identidad (p. 10).

Por lo tanto, «descentrarse» es una competencia intercultural fundamental, supone contrastar nuestros puntos de vista con los de otras personas o grupos para poder matizarlos. Cada opinión particular es relativa y tan sólo toma forma verdaderamente tras la interacción con otras personas o grupos. Para comprender al otro hay que observarse a uno mismo y ser capaz de distanciarse del sistema de valores propio para comprenderle desde el suyo.

\section{A modo de conclusión}

Como hemos tratado de reflejar en este artículo, el desarrollo de la competencia intercultural puede ser un enfoque que facilite el diálogo entre las diferentes interpretaciones de la salud (disease, illness, sickness); que integre saberes y prácticas de los distintos sectores que intervienen en la construcción de los sistemas de atención (popular, profesional, folk); y que contribuya a la coproducción de saberes en la atención sociosanitaria. En el caso específico de las drogodependencias, los cinco procesos de competencia institucional propuestos y los cuatro de competencia profesional pueden contribuir al desarrollo de políticas y a la construcción de relaciones de ayuda y/o terapéuticas mejor adaptadas, más próximas, más centradas en la persona y, por todo ello, más eficaces y eficientes. En ese sentido, un enfoque intercultural como el sostenido en este artículo es posible y, sobre todo, deseable.

Aunque la competencia intercultural es fundamental en todo tipo de profesiones y ámbitos, desde nuestro punto de vista, entronca de una manera especialmente adecuada con el Trabajo Social. Los procesos de competencia institucional y profesional propuestos son coherentes con principios fundamentales de esta disciplina, como el enfoque centrado en la persona, el abordaje integral, la participación, la adaptación a los contextos, o la construcción de vínculos desde la cercanía y la confianza. Además, su desarrollo puede servir para recobrar la proximidad que, desde algunos sectores del Trabajo Social, se ha debilitado tras determinados procesos de burocratización. 


\section{Referencias bibliográficas}

Arza, J. y Carrón, J. (2014). La influencia de los sistemas o modelos de creencias en la evolución de las políticas sobre drogodependencias. InfoNOVA, 1. Recuperado de: http://dianova.es/pdf/revista_infonova.pdf

Arza, J. y Carrón, J. (2014). Las estrategias de proximidad y centradas en la persona como alternativa a la fragmentación en la atención. Documentos de Trabajo Social, 54.

Boladeras, M. (coord.) (2007). Orientaciones sobre la diversidad cultural y la salud. Barcelona: Generalitat de Catalunya. Recuperado de: http://comitedebioetica.cat/wp-ontent/uploads/2012/09/diversidad_cultural.pdf

Canals, J. (2004). La importancia de las dimensiones culturales en la asistencia y en la promoción de la salud. Revista de Servicios Sociales y Política Social, 65, 9-20.

Cohen-Emerique, M. (2000). El acercamiento intercultural. Sevilla: Cuadernos de Formación de Sevilla Acoge.

Comas, D. (1986). Uso de drogas: del paradigma lewiniano al nuevo rol de las expectativas simbólicas. Jano Medicina y Humanidades, 713, 65-81.

Comas, D. (2005). La trayectoria vital y la experiencia terapéutica de los adictos a drogas ilegales en Castilla-La Mancha. Toledo: Fundación para la Investigación Sanitaria en Castilla-La Mancha (FISCAM). Recuperado de: http://www.od.jccm.es/portalNuevo/admin/modulos/publicaciones/ pdf/86_8de4r1x1.pdf

Departamento Salud EE.UU. (2001). Nacional Standards for Culturally and Linguistically Appropriate Services in Health Care. Executive Summary en español. Washington, D.C.: Departamento Salud USA.

Fernández Liria, A. y Pérez Sales, P. (coords.) (2011). Instrumento para la valoración de la competencia intercultural en la atención en salud mental. Hacia la equidad en salud. Madrid: Ministerio de Sanidad, Política Social e Igualdad. Recuperado de: http://www.mspsi.gob.es/profesionales/saludPublica/prevPromocion/promocion/desigualdadSalud/docs/Competencia_Intercultural_a ccesible.pdf

Kleinman, A. (1980). Patiens and healers in the context of culture. An Exploration of Borderland between Anthropology, Medicine and Psychiatry. Berkeley: University of California Press.

Lillo Crespo, M., Vizcaya Moreno, F., Domínguez Santamaría, J.M. y Galao Malo, R. (2004). Investigación cualitativa en enfermería y competencia cultural. Index Enfermería,13(44-45),47-50. Recuperado de: $\mathrm{http} / /$ scielo.isciii.es/scielo.php?script=sci_arttext\&pid=S1132-12962004000100010

Malik, B. (2009). Desarrollo de competencias interculturales en orientación. (s.n). Recuperado de: $\mathrm{http} / / /$ www.uned.es/centrointer/Competencias_interculturales.pdf

Osorio-Merchán, M.B. y Lucero, A. (2008). Competencia cultural en salud: necesidad emergente en un mundo globalizado. Index Enfermería, 17(4), 266-270. Recuperado de: http://scielo.isciii.es/ scielo.php?script $=$ sci_arttext\&pid $=$ S1132-12962008000400010

Qureshi Burckhardt, A., Revollo, H.W., Collazos, F., Visiers Würth, C. y El Harrak, J. (2009). La mediación intercultural sociosanitaria: implicaciones y retos. Norte de Salud Mental, 35,56-66.

Romaní, O. (2002). Criterios de prevención: un debate necesario. En Martín Hopenhayn (comp.), Prevenir en drogas: enfoques integrales y contextos culturales para alimentar buenas prácticas. Santiago de Chile: CEPAL Naciones Unidas.

Romaní, O. y Rekalde, A. (2002). Los medios de comunicación social ante las drogas: un análisis crítico. Observatorio Vasco de Drogodependencias. Vitoria-Gasteiz: Gobierno Vasco.

Vázquez, O. (2002). Trabajo social y competencia intercultural. Revista Portularia,2, 125-138. Recuperado de: http://rabida.uhu.es/dspace/handle/10272/140

Vilà, R. (2006). La dimensión afectiva de la competencia comunicativa intercultural en la educación secundaria obligatoria: escala de sensibilidad intercultural. Revista de Investigación Educativa, 24(2), 353-372. Recuperado de: http://revistas.um.es/rie/article/viewFile/96891/93071 